\title{
EL PODER DE POLICÍA EN LA HISTORIA CONSTITUCIONAL ESTADOUNIDENSE
}

\author{
SANTIAGO LEGARRE*
}

\section{A LA MEMORIA DE GERMÁN BIDART CAMPOS}

RESUMEN: Trátase en este trabajo de mostrar la formación y evolución del concepto de poder de policía en los Estados Unidos de América, en el cual el poder de policía tiene su antecedente inmediato en la idea de policía que fue importada de Europa a partir de ideas y tradiciones que arrancan del common law y del Derecho continental. Luego trata cómo el concepto policía derivó en policía interna para llegar al moderno concepto de poder de policia. Finalmente se bace una referencia a la influencia de estas concepciones en el Derecho público argentino.

Palabras clave: Policía - Poder de Policía - Delegación - Poderes delegados.

ABSTRACT: The present paper intends to explain the formation and evolution of the concept of power of police in the United States of America, in which power of police has its immediate antecedent in the idea of police imported from Europe as of the ideas and traditions that start with the Common Law and the Continental Law. It then deals with how the concept police drew into internal police to reach the modern concept of power of police. It finally makes a reference to the influence of these conceptions in the Argentinean Public Law.

Key words: Police - Power of Police - Delegation - Delegated powers.

SUMARIO: A) Nace la idea de "policía". B) La idea de policía en los Estados Unidos. C) Una cuestión de nombres: de "policía" a "poder de policia". D) El poder de policía, broad y narrow. E) Conclusión.

Director y profesor del Departamento de Filosofía del Derecho y Derecho Constitucional de la Universidad Austral, Argentina. Visiting Professor, Paul M. Hebert Law Center, Louisiana State University. Este trabajo está parcialmente basado en una tesis presentada en la Universidad de Oxford para el título de Master of Studies in Legal Research. Agradezco los valiosos comentarios y aportes de Juan Cianciardo, Manuel García-Mansilla y Rodrigo Ruiz Esquide. Artículo recibido el 17 de marzo de 2005. Aceptado por el Comité Editorial el 20 de mayo de 2005. 


\section{A) NACE LA IDEA DE "POLICÍA"}

Este trabajo tiene por finalidad mostrar la formación y evolución del concepto de poder de policía en los Estados Unidos de América. En este país el poder de policía tiene su antecedente inmediato en la idea de policía que fue, como veremos, importada de Europa.

Hoy entendemos por "policía" el cuerpo de oficiales que velan por la seguridad pública y trabajan en la persecución del crimen. No siempre fue así. En la España del siglo XIV, "policía" significaba "política"1; cuatro siglos más tarde connotaba "la buena orden que se observa y guarda en las Ciudades y Repúblicas, cumpliendo las leyes u ordenanzas, establecidas para su mejor gobierno"2. En Inglaterra la semántica de la policía transpiraba aires parecidos, tomados prestados de Francia ${ }^{3}$. De acuerdo con el Oxford English Dictionary, a comienzos del siglo XVIII se entendía por "police" la regulación, disciplina y control de una comunidad, la administración civil y el orden público ${ }^{4}$. Según esta autorizada fuente, el primer uso oficial de la palabra "police" en Gran Bretaña tuyo lugar en 1714 cuando la Reina ANA nombró comisionados de policía (Commisioners of Police) para la administración general interna de Escocias 5 .

Escocia, por lo demás, tuvo mucho que ver con el desarrollo de la idea de policía en las islas británicas. Allí nació en el siglo XVIII la categoría de "ofensas contra la policía", de carácter misceláneo y amplios alcances. Se trata de crímenes que no se dirigen contra personas particulares sino contra el Estado y el buen gobierno del país. Los autores escoceses de este período -principalmente Lord KAMES y John ERSKINEdan unos pocos ejemplos: las reglas para la prevención de incendios, las relacionadas con la prostitución y con la presencia en tabernas después de ciertas horas, las ofensas de "forestalling" (comprar grandes cantidades de cereal para después venderlo a un precio mayor cuando haya escasez) y de vagancia ${ }^{6}$. Veremos más adelante la influencia que esta caracterización de

Corominas, J., y Pascual, J. A.: Diccionario Crítico Etimológico Castellano e Hispánico, Gredos, Madrid, 1981, Vol. IV, sub voce "político" y su derivado "policía".

2 Diccionario de Autoridades: edición facsímil de la edición original de 1726-1737, Gredos, Madrid, 1976, Vol. III, s.v. "policía".

Chambers Dictionary of Etymology: Chambers, Edinburgo, 1988, s.v. "police".

Oxford English Dicrionary: segunda edición, Clarendon Press, Oxford, 1989, s.v. "police", significado 3.a.

Ibidem.

6 KAMES, LORD H. H.: Statute Law of Scotland Abridged. With Historical Notes, impresa por Sands et al. para A. Kincald y A. Donaldson, Edinburgo, 1757, p. 271; ErSKINE, J.: An Institute of the Law of Scotland, impresa por J. Bell en Addison's Head, Edinburgo, 1773 , n. 16, p. 705 y nn. 38-39, p. 714. Véase también, MACKEnZIE, Sir G.: The Institutions of the Law of Scotland, cuarta edicion, impresa por J. Watson para J. Vallange, Edinburgo, 1706 , p. 223. 
la policía tuvo en BLACKSTONE, cuyo influjo, a su vez, fue decisivo en los Estados Unidos.

Adam SMITH también contribuyó a la formación del concepto de policía en la Escocia ilustrada ${ }^{7}$, aunque su caracterización económica de la policía se tornó pronto obsoleta ${ }^{8}$.

En la Europa continental el primer teórico de la policía fue el jurista suizo Emmerich de VATTEL, cuyo Droit de Gens fue traducido al inglés en 1759, al año siguiente de su publicación original. Esta circunstancia facilitó la difusión en el mundo de habla inglesa de un libro que en Estados Unidos habría de ser considerado desde el principio un oráculo del derecho?.

Para VATTEL, el soberano debe cuidar de la nación "como un padre tierno y sabio, y como un administrador fiel" ${ }^{10}$. En cuanto padre, debe ocuparse de la verdadera felicidad de la nación, que constituye uno de los principales objetos de un buen gobierno ${ }^{11}$. A este objeto del gobierno VATTEL lo llama "policía"12:

7 Smith trata la policía en sus Lectures on Jurisprudence, impartidas en la Universidad de Glasgow entre 1762 y 1764 , y publicadas por primera vez en 1896 . Véase, Smith, A.: Lectures on Jurisprudence, edición a cargo de R. L. Meek, D. D. Raphael y P. G. Stein, Clarendon Press, Oxford, 1978, n. 5, p. 398 y n. 203, p. 486.

Oxford English Dictionary: s.v. "police", significado 3.b.

9 Sobre la autoridad del libro de Vattel en los Escados Unidos, véase CrosskeY, W.W. Politics and the Constitution in the History of the United States, The Universicy of Chicago Press, Chicago, 1953, Vol. I, p. 147; FENwICK, C. G.: "The Authority of Vatrel" (1913) 7 The American Political Science Review 395; Kelly, J.M.: A Short History of Western Legal Theory, Clarendon Press, Oxford, 1992, ps. 299-300. Véase también el "oral argument" del caso Gibbons v. Ogden, 9 Wheat. 1, 66 (1824), con cita de Vattel.

10. VATtel, E. de: The Law of Nations or Principles of the Law of Nature: applied to the Conduct and Affairs of Nations and Sovereigns, Newbery et al., Londres, 1759-1760, n. 42, p. 21. En otro trabajo he ensayado una incerpretación de los texcos de Vattel y de Blackstone sobre la policía desde la perspectiva de la analogía entre rey y padre que ambos aceptan. Legarre, S.: Poder de Policia y Moralidad Pública. Fundamentos y Aplicaciones, Ábaco, Buenos Aires, 2004, pp. 71-92.

11 Para Vattel el fin u objeto de la sociedad civil, constitutivo de su perfección, es procurar en general todo lo que constituya la felicidad, tanto de ella misma como de sus miembros individuales. Vattel, E. de: Law of Nations..., cit., n. 17, p. 13.

12 La palabra original francesa es police. En inglés fue traducida como polity (en la primera traducción de 1759-1760 y en la de 1793) y como police (en la traducción de 1797, editada por Joseph Chitty y publicada recién en 1834). Estas palabras (polity y police) se usaban intercambiablemente en el siglo XVIII: véase, CrOsSKEY, W. W.: Politics and the Constitution..., cit., Vol. 1, p. 149. Las citas incluidas en este trabajo están basadas en el original francés (Le Droit de Gens, ou Principes de la Loi Naturelle, appliqués à la Conduite et aux Affaires des Nations et des Souverains, Londres y Leiden, 1758) y en la primera edición inglesa, pero en todos los casos remiten a la paginación de esta última. Para una bibliografía completa de las diferentes ediciones del libro de Vattel en diversas lenguas, véase Lapradelle, A. de: "Introduction", en Brown Scott, J. (ed.), The Classics of International Law. Le Droit des Gens, ou Principes de la Loi Naturelle, appliqués à la Conduite et aux Affaires des Nations et des Souverains, by E. de Vattel. Volume III, Carnegie Institution of Washington, Washington, 1916, pp. Ivi-lix. 
La policía consiste en la precaución que deben tener el príncipe y los magistrados para preservar todo en orden. Sabias regulaciones deben prescribir todo lo que contribuya a la seguridad, utilidad y conveniencia públicas, y los que tienen en sus manos autoridad nunca se preocuparán demasiado de que aquellas sean tenidas en mira. Mediante una sabia policía el soberano acostumbra a la gente al orden y a la obediencia y preserva la paz, la tranquilidad y la concordia entre los ciudadanos ${ }^{13}$.

¿Qué tipo de regulaciones tiene en mente VA'TTEL cuando se refiere a la policía? Primero, da el ejemplo de la prohibición del duelo ${ }^{14}$, un lugar común entre las normas de policía en tiempos venideros. Más adelante menciona las limitaciones a la disposición de bienes por parte de los pródigos: el soberano, como un buen padre, debe poner los medios para evitar la ruina económica de sus "hijos pródigos"15. Finalmente, en el contexto de los límites de la propiedad privada afirma "que los particulares no son tan libres en la economía o el gobierno de sus bienes como para no estar sujetos a las regulaciones de policía dictadas por el soberano"16. Por ejemplo, si en un país con escasez de maíz sobreabundan las plantaciones de viña el soberano puede prohibir que se planten viñas en terrenos apropiados para el cultivo de maíz, porque en este caso el bienestar público y la seguridad del Estado están en juego ${ }^{17}$.

En Inglaterra, quien más se ocupó del concepto de policía en el siglo XVIII fue Sir William BLACKSTONE. Sus ideas sobre la materia, como ya se dijo, tuvieron una gran repercusión en los incipientes Estados Unidos y por eso son también importantes a los fines de este estudio ${ }^{18}$. BLACKSTONE trata la policía en dos partes de sus Commentaries on the Laws of England, publicados entre 1765 y $1769^{19}$ : en el Libro Primero, al tratar

VATTEL, E. de: Law of Nations..., cit., n. 174, pp. 76-77, énfasis añadido.

Ibídem, n. 175, p. 77.

Ibídem, n. 254, p. 104.

Ibídem, n. 255, p. 104, énfasis agregado.

Otros ejemplos de medidas de policía relacionadas con la propiedad privada: "Cuando alguna razón de importancia suficiente lo requiere, el soberano o el magistrado pueden obligar a un particular a vender todas las provisiones que excedan lo necesario para la subsistencia de su familia, y fijar el precio. La autoridad pública puede y debe desalentar los monopolios y suprimir toda práctica tendiente a aumentar el precio de las provisiones...". Tanto el ejemplo del texto como los transcriptos precedentemente pueden encontrarse en VATTEL, E. de: Law of Nations,.., cit., n. 255, p. 104.

18 Sobre la influencia inmediata de Blackstone en los Estados Unidos, véase Nolan, D. R.: "Sir William Blackstone and the New American Republic: a Study of Intellectual Impact" (1976) 51 New York University Law Review 731, p. 737.

19. Sigo aquí la primera edición de 1765-1769 dado que las secciones relevantes no sufrieron modificaciones significativas en las restantes ediciones realizadas en vida de Blackstone, la última de las cuales fue la octava, de 1778. 
de la prerrogativa del rey ${ }^{20}$; y en el Libro Cuarto, al tratar de las injurias públicas (public wrongs).

Entre las prerrogativas reales recogidas en el Libro Primero y relacionadas con los asuntos internos se encuentran las que derivan de la condición del rey como árbitro del comercio doméstico. En ejercicio de dicha potestad puede establecer:

[M]ercados públicos, o lugares de compra y venta, como ferias y mercados, con los peajes correspondientes. Aquellos solamente pueden abrirse por permiso del rey, o por un uso y prescripción extensos e inmemoriales, que presuponen un tal permiso. La limitación de estos puntos de reunión al tiempo y lugar más convenientes para el vecindario, forma parte de la economía o policía doméstica; la cual, considerando el reino como una familia grande y al rey como su señor [master], comporta su derecho de disponer y ordenar como a él le plazca $^{21}$.

El poder de dar curso legal a la moneda y la regulación de las pesas y medidas constituyen otros ejemplos de prerrogativas reales sobre el comercio. BLACKSTONE afirma que las pesas y medidas "[d]eben ser las mismas en todo el reino para beneficio del público"22. La idea de policía aparece así, nuevamente, relacionada con el dominio público. Y queda claro que policía es sinónimo de administración civil o doméstica.

Este mismo sentido de "policía" se halla presente cuando sobre el final de su obra, en el Libro Cuarto, BLACKSTONE aborda el Derecho penal. Las injurias públicas (public wrongs), afirma, se dividen en dos. En primer lugar, están "aquellos crímenes y contravenciones que afectan más especialmente el bienestar general [common-wealth], o la policía pública del reino" 23 . En segundo término, "aquellos peculiarmente dirigidos contra la vida y la seguridad de sujetos privados" 24 , como el homicidio y la violación. Los delitos contra la policía pública ofenden al rey "en su

20 Ernst Freund, el autor estadounidense más influyente del siglo XX en materia de poder de policía, subraya la relación entre prerrogativa real y policía al denominar a la primera "poder de policía real". FreUND, E.: Standards of American Legislation. An Estimate of Restrictive and Constructive Factors, The University of Chicago Press, Chicago, 1917, p. 38 .

21 BLACKSTONE, SiR W.: Commentaries on the Laws of England, edición facsímil del original de 1765-1769, University of Chicago Press, Chicago, 1979, Vol. I, p. 264, énfasis añadido. Crosskey explica que "economía" y "policía" eran usados por Blackstone como sinónimos. Crosskey, W.W.: Politics and the Constitution..., cit., Vol. I, p. 148. como las pesas y medidas son por naturaleza arbitrarias e indefinidas, es por tanto útil que sean reducidas a alguna regla o estándar fijos". 
condición de paterfamilias de la nación; a quien pertenece en virtud de su oficio real proteger a la comunidad, y a cada uno de los individuos que la integran, contra todo tipo de violencia dañosa mediante el dictado de leyes $[\ldots]^{\prime 25}$. Pareciera que la dimensión pública es más inmediata en el caso de este tipo de faltas. Si bien en las del segundo grupo también se da una afectación del interés público, esta viene mediada por el perjuicio que originalmente sufre un "sujeto privado" (por ejemplo, la víctima de un homicidio). Ello no ocurre con las ofensas del primer grupo, que atentan más especialmente contra el bienestar del reino -la policía públicay contra el rey.

Los crímenes y contravenciones que afectan más especialmente la cosa pública (common-wealth) pueden dividirse, según BLACKSTONE, en cinco especies: "ofensas contra la justicia pública, contra la paz pública, contra el comercio público, contra la salud pública, y contra la economía o policía públicas" 26 . Obsérvese que si bien antes el autor había usado como sinónimos "common-wealth" y "policía pública" 27 , al ofrecernos esta clasificación enseña que las ofensas contra la policía (o economía) pública son una especie de las ofensas que afectan más especialmente la cosa pública (common-wealth). Detrás de este uso flexible del lenguaje podemos adivinar que hay un sentido más amplio de policía, en el cual se identifica con la cosa o bienestar públicos (common-wealth) en toda su extensión y, por lo tanto, incluye la justicia, la paz, el comercio y la salud públicas; y otro sentido más estrecho y preciso, que engloba solamente algunas de las materias que hacen al common-wealth. ¿Cuáles? Dejemos que hable BLACKSTONE:

Por la policía y economía públicas significo la debida regulación y orden doméstico del reino: por medio del cual los individuos del estado, como miembros de una familia bien gobernada, están obligados a conformar su conducta general a las reglas de corrección, decoro y buenas maneras [good manners]; y a ser decentes, industriosos $\mathrm{e}$ inofensivos en sus respectivos puestos ${ }^{28}$.

Nuevamente sobresale la dimensión pública de la policía, ya que se trata de "todos los crímenes que especialmente afectan a la sociedad pública y no estén comprendidos en ninguna de las cuatro especies precedentes" 29 ; o, en otras palabras, de "ofensas contra el orden público y el régimen

\footnotetext{
25 Ibidem.

26 Ibídem, Vol. IV, p. 128.

27 Véase la ya citada expresión "crímenes y contravenciones que afectan más especialmente el bienestar general [common-wealth], o la policía pública del reino". BLACKSTONE, SiR W.: Commentaries..., cit., Vol. IV, p. 127. 
económico del estado" 30 . Por ello, como en Escocia, este título de ofensas debe ser necesariamente "misceláneo" 31 y, cabe agregar, residual, lo cual excluye una definición de contornos claros. La referencia al orden público para explicar la policía aporta cierta luz pero no debe olvidarse que en el common law "orden público" tiene un significado un tanto más estrecho que en el derecho continental. Mientras que en este último el "orden público" va de la mano de la moral pública y las buenas costumbres ${ }^{32}$, en el derecho anglosajón significa ausencia de desorden, esto es, paz, tranquilidad, seguridad públicas ${ }^{33}$-bienes todos estos, también englobados en la noción civilista de "ordre public" 34 - Sin desconocer las connotaciones más amplias que la noción supone en los ordenamientos de raíz francesa, no puede negarse, a la luz de los textos analizados, que en el common law las good manners tampoco son ajenas al public order que el rey tutela mediante la policía.

BLACKSTONE ensaya una enumeración de los crímenes contra la policía pública en el capítulo XIII del Libro IV, el mismo capítulo en el que trata de los crímenes contra la salud pública. Esto último es digno de señalamiento pues de las cinco especies de ofensas contra el commonwealth, las tres primeras (justicia, paz y comercio) merecen tratamiento separado en los tres capítulos precedentes; solo a estas dos (salud y policía) se las trata en un mismo capítulo. Ello no habría de pasar inadvertido a los ojos de la historia ya que la salud pública será, con el paso del

30 Ibídem, Vol. IV, p. 167; énfasis añadido.

31 Ibídem, Vol. IV, p. 162.

32 Cfr., por ejemplo, en la Argentina el artículo 19, primera parte, de la Constitución Nacional ("Las acciones privadas de los hombres que de ningún modo ofendan al orden y a la moral pública, ni perjudiquen a un tercero, están solo reservadas a Dios, y exentas de la autoridad de los magistrados") y el artículo 21 del Código Civil ("Las convenciones particulares no pueden dejar sin efecto las leyes en cuya observancia estén interesados el orden público y las buenas costumbres").

33 Obsérvese, solo a título de ejemplo de lo dicho en el texto, la siguiente afirmación de Tiedeman en el prefacio de su importante tratado sobre limitaciones al poder de policía en los Estados Unidos, escrito a fines del siglo XIX: "[...] Por el contrario, la esfera de acción gubernamental se hallaba confinada dentro de límites estrechos por la popularización de la así llamada doctrina del laissez-faire, que niega al gobierno el poder de hacer más que lo necesario para proveer el orden público (public orderł y la seguridad personal mediante la prevención y castigo de crímenes y transgresiones". TIEDEMAN, C.G.: A Treatise on State and Federal Control of Persons and Property in the United States considered from both a civil and criminal standpoint, The F. H. Thomas Law Book Co., St. Louis, Missouri, 1900, p. vi. Es decir que el aseguramiento del public order era compatible con un estado mínimo. Pareciera que no podría decirse otro tanto del orden público al estilo francés.

34 Véase, Finnis, J.M.: Ley Natural y Derechos Naturales, Abeledo-Perrot, Buenos Aires, 2000, ps. 243-246. Finnis considera notoria la diferencia de significado entre la expresión inglesa public order y la francesa ordre public. Ibídem, p. 257. 
tiempo, uno de los bienes corrientemente incluidos entre los objerivos del poder de policía ${ }^{35}$.

He aquí, pues, el listado de ofensas contra la policía pública ${ }^{36}$ :

- matrimonios clandestinos

- bigamia

- vagancia (incluye el problema de los soldados y marineros ociosos, y de los egipcios o gitanos [Egyptians or gypsies])

- lujo o suntuosidad

- juegos de azar (gaming ${ }^{37}$ )

- cacería de ciertos animales y aves salvajes genéricamente llamados en inglés game (por ello las leyes que prevén esta ofensa se llaman game laws $^{38}$ )

- molestias públicas (common nuisance)

Este último tipo de ofensas contra la policía pública llamado common nuisance, expresión que carece de un equivalente exacto en nuestra lengua $^{39}$, requiere un párrafo aparte ${ }^{40}$. BLACKSTONE la define así: "la realización de una cosa que molesta a todos los súbditos del rey, o la negligencia en la realización de una cosa que requiere el bien común"41. A

35 Más aún, los ejemplos de ofensas contra la salud pública que da Blackstone -como la venta de provisiones insalubres - son semejantes a algunas de las "regulaciones de policía" que enumera uno de los primeros constitucionalistas de los Estados Unidos, James Kent. KENT, J.: Commentaries on American Law, undécima edición, editada por G. F. Comstock, Little, Brown, and Company, Boston, 1866, Vol. II, n. 340, p. 415.

Véase esta enumeración en BLACKSTONE, SIR W.: Commentaries..., cit., Vol. IV, pp. 163-167.

57. El verbo antiguo gaming, usado por Blackstone, derivó en gambling, que modernamente se usa para designar la participación en juegos de azar. Ayto, J.: Dictionary of Word Origins, Arcade Publishing, Little, Brown, and Company, New York, 1990, p. 248, s.v. gamble y game.

Cabanellas de las Cuevas, G., y Hoague, E. C.: Diccionario Juridico Inglés-Español, Heliasta, Buenos Aires, 1993, Vol. I, p. 275, s.v. game y game laus.

39 Cabanellas de las Cuevas y Hoague definen common nuisance como "molestia pública. Acrividad o conducta que afecta al público en general, produciéndole un daño o molestia". Cabanellas de las Cuevas, G., y Hoague, E. C.: Diccionario..., cit., Vol. I, p. 123, s.v, common nuisance. La expresión tiene un significado parecido al de public nuisance: "conducta, condición o circunstancia que causa un daño al interés público, sea por perjudicar o impedir el uso de bienes públicos o por ocasionar molestias al público". Ibídem, Vol. 1, p. 506, s.v. public nuisance.

10 El historiador de la Universidad de Chicago William J. Novak, en un trabajo que ilumina muchos aspectos de esta cuestión, destaca la enorme importancia del derecho de las nuisances (law of nuisance) en la conformación de la "well-regulated society", frase que caracteriza bien, a su juicio, a la sociedad estadounidense del siglo XIX en la que cristalizaron ideas como las sostenidas por Blackstone. Véase NOVAK, W.J.: The People's Welfare. Law and Regulation in Nineteenth-Century America, The University of North Carolina Press, Chapel Hill, 1996, passim.

i1 Blackstone, SiR W.: Commentaries..., cic,, Vol, IV, p. 167. 
diferencia de la private nuisance-interferencia con el goce de un inmueble determinado-, la common nuisance "molesta a toda la comunidad en general, y no meramente a alguna persona particular" 42 . Las common nuisances constituyen todo un subgénero de ofensas contra la policía pública. BLACKSTONE da los siguientes ejemplos:

- molestias en la vía pública, así como en puentes y ríos públicos

- comercio e industrias ofensivas (cuando son perjudiciales para el público y no meramente para una persona en particular)

- albergues inmorales (disorderly inns ${ }^{43}$ ) y cervecerías (ale-bouses)

- loterías

- fabricación y venta de fuegos artificiales ${ }^{44}$

No me extenderé aquí en el sentido y alcance de la idea de policía, tal como la encontramos en estos autores dieciochescos ${ }^{45}$. Queda claro que "policía" significaba algo muy distinto de lo que significa hoy y que se trataba de un concepto directamente vinculado con lo público. Sin tener una definición clara, abarcaba realidades cercanas tanto a lo que después se llamó moralidad pública como a la seguridad, sin dejar de incluir regulaciones de tipo económico. Veremos ahora cómo la policía cruzó el Atlántico, se instaló en Estados Unidos y se transformó en el poder de policía.

\section{B) LA IDEA DE POLICÍA EN LOS ESTAdos UNIDOS}

Ya se adelantó el impacto de las ideas de BLACKSTONE en el despertar de la experiencia institucional estadounidense. Esta influencia se hizo sentir especialmente en el tema que estamos tratando. Algunos estados de la Unión incluso incorporaron a su legislación doméstica su clasificación de las ofensas en cinco especies, dedicando un título separado a los crímenes contra la policía ${ }^{46}$. En una nota a una edición norteamericana de los Commentaries, HAmmond afirma que el influjo de BlaCKSTONE "es más evidente que en ningún otro punto en los importantes efectos pro-

42 Ibidem.

43 Cabanellas de las Cuevas y Hoague definen disorderly bouse como "casa en que se realizan conductas contrarias al orden y a la tranquilidad pública". CABANELLAS DE LAS CUEVAS, G., y HoAgue, E.C.: Diccionario..., cit., Vol. I, p. 193, s.v. disorderly house. Blackstone, SiR W.: Commentaries..., cit., Vol. IV, p. 167.

is Remito al lector interesado a LegarRe, S.: Poder de policia..., cit., pp. 83-92.

46 La palabra "policía" aparece por primera vez como una división dentro de una ley en los Revised Statutes of New York (1829). En 1836 también la adoptó Massachusetts y, con posterioridad, otros siete estados. Freund, E,, The Police Power. Public Policy and Constitutional Rights, reimpresión del original de 1904, Arno Press, New York, 1976, n. 2, p. 2 , nota 2 . 
ducidos sobre la interpretación de la constitución federal y las constituciones estatales en virtud del reconocimiento [de la categoría de ofensas contra la policía] como un poder gubernamental distintivo, no abarcado por el dominio eminente ni sujeto a sus limitaciones" 47 .

Como ya se dijo, VATTEL también tuvo una influencia destacada en los Estados Unidos. Crosskey demuestra en su libro Politics and the Constitution in the History of the United States que las ideas de VATTEL sobre la policía eran muy conocidas en la Norteamérica del siglo XVIII. Sin perjuicio del carácter controvertido de la obra de CROSSKEY ${ }^{48}$, parece estar fuera de duda que para los "padres fundadores" de la Constitución estadounidense "policía" significaba algo semejante a lo que significaba para VATTEL (y también para BLACKSTONE) ${ }^{49}$.

Además de este significado, al que se ha hecho referencia en la sección precedente de este trabajo, los términos "police" y "polity" (sinónimos en el siglo XVIII y traducibles ambos como "policía") tenían otro sentido en Norteamérica, especialmente cuando iban acompañados del adjetivo "interno" (como en "policía interna"). Este significado puede observarse, por ejemplo, en los debates que tuvieron lugar durante el Primer Congreso Continental de $1774^{50}$. Allí, la expresión fue usada varias veces para contrastar la policía interna de las colonias con los poderes de Gran Bretaña ${ }^{51}$, y los representantes de las colonias separatistas defendieron el derecho de los estados nacientes a una "legislación exclusiva [...] en todos los casos de tributación y policía interna"52. "Policía interna" en este contexto tenía un significado amplio; connotaba, en efecto, un ámbito de soberanía local o doméstica que implicaba una supuesta limitación para el parlamento británico. Las colonias estaban dispuestas a consentir "a la operación de actos del parlamento británico tales que estén bona fide limitados a la regulación de nuestro comercio externo"53. Lo demás, es decir, tributación y policía interna, quedaba reservado a las colonias.

\footnotetext{
47 Blackstone, SiR W.: Commentaries on the Laws of England, octava edición, W.G. HAMMOND, Bancroft-Whitney Company, San Francisco, 1890, Vol. IV, p. 217. titutional Law, tercera edición, The Foundation Press, New York, 2000, Vol. I, Pp. 6162 y nota 52 .

49 Crosskey aporta considerable evidencia en este sentido. CrossKeY, W. W.: Politics and the Constitution..., cit., Vol. I, pp. 147-152; Vol. II, p. 1179.

50 El Primer Congreso Continental de 1774 era el cuerpo de delegados que representaban a las colonias que más tarde se convertirían en los Estados Unidos de América.

51 Véase, por ejemplo, el plan de unión entre Gran Bretaña y Norteamérica presentado por Joseph Galloway en septiembre de 1774. Journals of the Continental Congress (1774-1789), edición a cargo de W.C. FORD et al., Government Printing Office, Washington, 19041937, Vol. I, p. 49.

52 Journals..., cit., Vol. I, p. 68

53 Ibidem, Vol. I, pp. 68-69.
} 
Después de la Declaración de Independencia de los Estados Unidos de América en 1776, que no contiene una referencia explícita a la policía interna, esta preocupación reaparecería en los Articles of Confederation adoptados por los trece nuevos estados en 1781. El artículo segundo declaraba que cada uno de los estados confederados retenía su soberanía, libertad e independencia, así como todo poder, jurisdicción y derecho, que no fuera expresamente delegado por la confederación a los Estados Unidos reunidos en el congreso continental ${ }^{54}$. Aunque esta vez no se usó el término "policía", la soberanía aludida era sin duda la "policía interna" del Primer Congreso Continental. La redacción adoptada en un borrador anterior de este artículo, que sí incluía la expresión "policía interna" para referirse a lo mismo, confirma esta interpretación ${ }^{55}$.

Inmediatamente después de la Declaración de Independencia, los nuevos estados separados de Gran Bretaña comenzaron a sancionar sus propias constituciones. Algunas de ellas incluían una "Declaración de Derechos" con una norma específica sobre "policía", que devino fórmula canónica. La siguiente era una expresión típica:

Solamente la gente de este Estado debe tener el exclusivo derecho de regular su gobierno y policía internos ${ }^{56}$.

El Preámbulo de la Constitución de New York (1777) se refería a "un tema tan importante como la necesidad de erigir y constituir una nueva forma de gobierno y policía interna que excluya todo tipo de jurisdicción, dominio y control extranjeros" 57 . De modo parejo, el Preámbulo de la Constitución de South Carolina (1776) consideraba que "para el bien de la gente debe establecerse, por común consentimiento, el origen y fin de todos los gobiernos para la regulación de la policía interna de esta colonia" 58 . El silencio de las demás constituciones originales puede suge-

Ibídem, Vol. IX, p. 908 y Vol. XIX, p. 214.

El borrador es de 1776; véase Journals..., cit., Vol. V, p. 547.

Arrículo 2 de la Declaración de Derechos de las Constituciones de Maryland (1776) y North Carolina (1776), énfasis agregado; artículo 3 de la Declaración de Derechos de la Constirución de Pennsylvania (1776); artículo 4 de la Declaración de Derechos de la Constitución de Vermont (1777). La Declaración de Derechos y Reglas Fundamentales de Delaware (1776) tenía una norma casi idéntica, la sección 4, que disponía: "Que solamente la gente de este estado tiene el exclusivo e inherente derecho de gobernar y regular su policía interna". La Declaración de Derechos de la Constítución de Massachussets (1780), artículo 4, se acerca a esa formulación sin usar la palabra "policía": "Solamente la gente de este estado riene el exclusivo derecho de gobernarse a sí misma como un estado libre, soberano e independiente $[\ldots]^{\prime \prime}$.

57 Constitución de New York (1777), Preámbulo, énfasis añadido.

58 Constitución de South Carolina (1776), Preámbulo, énfasis añadido. 
rir que el principio de la reserva de la policía a los estados era tan ampliamente aceptado que no necesitaba reconocimiento expreso $0^{59}$.

En este contexto, no causa sorpresa que el tema "policía" haya estado presente en las discusiones que condujeron a la Constitución de los Estados Unidos de 1787. En la convención constituyente federal hubo propuestas de incluir en la nueva constitución una "limitación de policía interna" al poder nacional. Roger SHERman, de Connecticut, propuso una resolución declarando que el gobierno federal no tendría derecho "a interferir con el gobierno de los Estados en ninguna materia de policía interna, que concierne solamente a los gobiernos de esos Estados y no compromete el bienestar general de los Estados Unidos"60. El Governeur MORRIS, de Pennsylvania, se opuso a SHERMAN argumentando que "la policía interna, como sería llamada y entendida por los Estados, debería ser infringida en muchos casos, como en el caso del papel moneda [...]"61. El punto de vista de MORRIS prevaleció y la resolución propuesta por SHERMAN fue rechazada. Una propuesta sustancialmente idéntica a la de SHERMAN fue hecha más tarde por el Comité de Detalle pero también se votó en contra de ella ${ }^{62}$. Por último, el 15 de septiembre de 1787 , SHERMAN reiteró su intento de obtener una limitación de policía interna, pero esta vez se encontró con la oposición de MADISON y volvió a fracasar ${ }^{63}$.

La decisión de la convención constituyente de no incluir una referencia a la policía interna de los estados en la constitución federal es un tanto sorprendente si se tiene en cuenta que dicha referencia hubiera significado continuidad con la posición defendida por las colonias unos años antes en el Congreso Continental y coherencia con las correspondientes normas de algunas constituciones estatales. Sin embargo, la omisión no debe ser malinterpretada. El silencio de la constitución federal debe entenderse en el contexto de la práctica precedente, que claramente confirmaba que los estados conservaban la policía interna. MADison lo expresó con lucidez en El Federalista, sin usar la palabra

59 Véanse las siguientes constituciones: New Hampshire (1776); New Jersey (1776); Virginia (1776); Georgia (1777). Rhode Island, otro de los trece estados originales, no tuvo una Constitución hasta 1842. Véase THORPE, F.N.: The Federal and State Constitutions, Colonial Charters, and other Organic Laws of the States, Territories, and Colonies now or heretofore forming the United Sates of America, Government Printing Office, Washington, 1909, passim.; STIMSON, E. J.: The Law of the Federal and State Constitutions of the United States. Boston Book Company, Boston, 1908, p. 125. Esto ocurrió el 17 de julio de 1787. The Records of the Federal Convention of 1787, 1937 Revised Edition, a cargo de M. Farrand, Yale University Press, New Haven, 1966, Vol. 2, p. 21.

61 Notes of Debates in the Federal Convention of 1787 Reported by James Madison, W.W. Norton, New York, 1966, p. 303.

62 Esto tuvo lugar el 22 de agosto de 1787. The Records... cit., Vol. 2, p. 367.

63 Notes of Debates..., cit., pp. 649-650. 
"policía" pero aludiendo significativamente a la "soberanía residual de los estados". La jurisdicción del gobierno federal, escribió, "se extiende únicamente a ciertos objetos enumerados y deja a los Estados una soberanía residual e inviolable sobre todos los demás"64. En sentido parecido, HAMILTON afirmaba:

La completa consolidación de los Estados dentro de una soberanía nacional implicaría la absoluta subordinación de las partes; y los poderes que se les dejaran estarían siempre subordinados a la voluntad general. Pero como el plan de la convención tiende solamente a conseguir una consolidación o unión parcial, los gobiernos de los Estados conservarían todos los derechos de la soberanía que disfrutaban antes y que no fueran delegados de manera exclusiva en los Estados Unidos por dicho instrumento ${ }^{65}$.

El principio formulado en estos pasajes de El Federalista se sigue de la enumeración de los poderes reconocidos al Congreso por el Artículo 1, Sección 8, de la Constitución de los Estados Unidos. Como afirmaría el Chief Justice MArshall en el famoso caso Gibbons v. Ogden (1824), "[la] enumeración presupone algo no enumerado" 66.

En tiempos modernos, la Corte Suprema estadounidense reafirmó esta idea, apoyándose en el Artículo 1, Sección 8, para sostener que "[la] Constitución [no otorga] al Congreso un poder de policía plenario que autorizaría la sanción de cualquier tipo de legislación"67. En todo caso es obvio que, como dice SCHEIBER, el significado histórico del concepto de policía "deriva del uso y la aplicación, no del lenguaje de la Constitución que nunca emplea el término"68. Aunque también es verdad que el lenguaje de la Enmienda Décima, introducida en 1789 como parte del Bill of Rights, captura bien la idea de policía (sin usar la palabra):

64 El Federalista: Fondo de Cultura Económica, México, 1994, No 39, p. 162. Sobre la contribución de Madison a la doctrina de los poderes enumerados véase, en nuestro medio, Garcia-Mansilla, M.J., y Ramirez Calvo, R.: "James Madison y la Constitución Nacional", en Homenaje a la Constitución Nacional de 1853 en el sesquicentenario de su sanción, Instituto Urquiza de Estudios Históricos-Universidad de Belgrano, Buenos Aires, 2003, pp. 149-151.

65 El Federalista: cit., $\mathrm{N}^{\circ} 32$, p. 127, énfasis en el original.

669 Wheat. 1, 195 (1824).

67 United States v. López, 514 U.S. 549, 566 (1995), voto de la mayoría.

68 SCHEiber, H.: "State Police Power", en LEVY, L., et al., Encyclopaedia of the American Constitution, MacMillan Publishing Company, New York, 1986, Vol. IV, p. 1744. Randy Barnett también considera los alcances del silencio de la constitución sobre la cuestión del ámbito apropiado del poder de policía. BARnETT, R.E.: "The Proper Scope of the Police Power", 79, Notre Dame Law Review, 429, 2004. 
Los poderes no delegados a Ios Estados Unidos por la Constitución ni prohibidos por esta a los Estados, quedan reservados respectivamente a los Estados o al pueblo ${ }^{69}$.

Esta enmienda refuerza la idea implícita en la enumeración del Artículo 1, Sección 8, y hay quien considera que la enmienda funciona como la principal base constitucional del poder de policía de los esta$\operatorname{dos}^{70}$, y quien entiende que "el poder de policía es uno de los poderes reservados a los Estados por la Enmienda Décima"71. En efecto, la Enmienda Décima refleja la "soberanía residual de los Estados", resultando claro que, bajo la nueva Constitución, la policía estaba en manos de los estados, considerados como gobiernos de jurisdicción general [governments of general jurisdiction]. El gobierno federal, por el contrario, era concebido como un gobierno de poderes delegados y enumerados, sin autorización constitucional para ejercer poderes de policía ${ }^{72}$.

\section{C) UNA CUESTIÓN DE NOMBRES: DE "POLICÍA" A "PODER DE POLICÍA"}

En los Estados Unidos la idea de policía recibió diversos nombres. Por ejemplo, James KENT, uno de los primeros constitucionalistas y profesor en la Universidad de Columbia, se refirió en 1826 al "poder de regulación"73, que, al decir de CORWIN, se convertiría a su tiempo en el "poder de policía" del derecho constitucional estadounidense ${ }^{74}$.

Fue la Corte Suprema de los Estados Unidos la que le cambió el nombre a la "policía", en el sentido de policía interna que explicamos en la sección anterior, y le dio la denominación nueva de "poder de policía". La incorporación de estas palabras al léxico del Tribunal fue gradual y

69 Constitución de los Estados Unidos, Enmienda Décima. Sobre la relación de esta enmienda con la doctrina de los poderes enumerados, véase GARCÍA-MANSILLA, M.J., y RAMÍreZ Calvo, R.: "James Madison...", cit., p. 149.

70 NOVAK, W.J.: The People's Welfare..., cit., p. 13.

71 Evans, L. B.: Leading Cases on American Constitutional Law, Callaghan \& Company, Chicago, 1925 , p. 1226.

72 "Todos reconocen que el gobierno [federal] es uno de poderes enumerados. El principio de que puede ejercer solo poderes que le sean reconocidos $[\ldots]$ es ahora universalmente admitido". McCulloch v. Maryland, 4 Whear. 316, 405 (1819). En la práctica este principio está en crisis, desde hace ya un tiempo, dado el incesante avance del gobierno federal. Véase George, R.P., "The Concept of Public Morality", 45, American Journal of Jurisprudence, 17, 2000, pp. 21-23.

73 KenT, J., Commentaries..., cit., Vol. II, n. 340, p. 415. Al respecto puede verse, en nuestro medio, Legarre, S.: "Los orígenes históricos del poder de policía", en El Derecho, 1999, 82-1048 y "El poder de policía en la bistoria, la jurisprudencia y la doctrina", en La Ley, 2000-A-999.

74 CORWIN, E. S.: Liberty against Government. The Rise, Flowering and Decline of a Famous Juridical Concept, Louisiana State University Press, Baton Rouge, 1948, p. 88. 
ocurrió de la mano de su primer Chief Justice, John MARSHALL ${ }^{75}$. Primero, en Dartmouth College v. Woodward ${ }^{76}$ (1819) el Tribunal afirmó que "los autores de la Constitución [framers] no quisieron limitar a los estados en la regulación de las instituciones civiles adoptadas para su gobierno interno"77. Enfatizamos el término "interno" por sus antecedentes en el constitucionalismo federal y local, y sus reminiscencias de lo "doméstico" en BLACKSTONE. Unos años después, la Corte incorporó por vez primera la palabra "policía" a su vocabulario en Gibbons v. Ogden 78 (1824), nuevamente en el contexto de la definición de los límites del federalismo: "el reconocido poder del estado de regular su policía, su comercio doméstico y de gobernar a sus propios ciudadanos puede capacitarlo para legislar en esta materia [el comercio] hasta cierto punto no desdeñable"79. Las leyes locales sub-examine [inspection laws] "forman una porción de esa inmensa masa de legislación que abraza todo lo que se encuentra en el territorio del estado y que no hubiera sido renunciado a favor del gobierno general" 80 .

Por fin, en Brown v. Maryland ${ }^{81}$ (1827) el Cbief Justice Marshall, que también había escrito para la Corte los dos fallos anteriores, habló por primera vez del "poder de policía" como un poder que "los estados incuestionablemente retienen y deben retener" 82 . CROSSKEY afirma que la frase "police power" es tan natural en cuanto tal, en vista de las nociones de la época, que resulta difícil creer que nunca hubiera sido utilizada antes de que el Chief Justice MARSHALL lo hiciera en este caso particular ${ }^{83}$. No obstante, de acuerdo con los mejores estudios sobre la materia Brown v. Maryland, constituye el punto de partida de esta terminología ${ }^{84}$. De esta manera, como cuando el capullo deja lugar a la mariposa, de la policía salió el poder de policía. La "frase combinada" -como se llamó años después al "poder de policía" 85 - no es otra cosa que un nuevo nombre para la vieja idea de policía. Walter COOK vio esto con claridad en un importante artículo publicado en 1907:

\footnotetext{
75 En un ensayo tan lúcido como extenso W. G. Hastings afirma que la idea de poder de policía le sobrevino al Chief Justice Marshall gradualmente. Hastings, W. G., "The Development of Law as Illustrated by the Decisions Relating to the Police Power of the State", en Proceedings of the American Pbilosophical Society, vol. XXXIX, 2000, n. 163, p. 363 .

764 Wheat. 518 (1819).

77 Dartmouth College, 629, énfasis añadido.

789 Wheat. 1 (1824).

79) Gibbons, 208, énfasis agregado.

80 Gibbons, 203.

$81 \quad 12$ Wheat. 419 (1827).

82 Brown v. Maryland, 443.

83 Crosskey, W.W.: Politics and the Constitution..., cit., Vol. II, p. 1305.

84 Véase Hastings, W.G.: “The Development of Law...", cit., p. 360

85. Ibidem, p. 366.
} 
¿Hay alguna conexión entre las dos frases? Creo que la hay, y que un estudio de la historia mostrará que la una fue sustituida por la otra, y que la frase más moderna "poder de policía" es hoy usada por nuestros tribunales en un sentido bastante idéntico al que los creadores [framers] de la Constitución le daban a la más temprana frase ["policía interna"] ${ }^{86}$.

El natural emerger semántico del poder de policía a partir de la policía es confirmado por las numerosas decisiones judiciales que hablan alternativamente de ambos para referirse a lo mismo. Así, en el celebrado voto para la Corte [opinion for the Court] del Justice FIELD en Barbier v. Connolly, primero se dice de una ordenanza local prohibitiva del lavado y planchado de ropa en lugares públicos a ciertas horas que es "puramente una regulación de policía dentro de la competencia de cualquier municipalidad que posea los poderes propios de esos cuerpos"87. Más adelante, para referirse a la misma ordenanza el Justice FIELD alude al ejercicio de los "poderes de policía" de las municipalidades ${ }^{88}$. Y en Mugler v. Kansas, el Justice HARLAN se refiere a las leyes que prohibían la manufactura y venta de bebidas alcohólicas primero como manifestaciones de "los poderes de policía del Estado"89, y después como "regulaciones de policía" 90 . No hace falta agobiar aquí con más ejemplos"1.

\section{D) EL PODER DE POLICÍA, BROAD Y NARROW}

En los casos que hemos repasado, de Brown v. Maryland en adelante, la frase "poder de policía" era usada como un sinónimo de lo que El Federalista denominaba la "soberanía residual de los estados", esto es, "esa autoridad general no entregada al Gobierno General y reservada individualmente por cada Estado para regular de la manera que lo considere apropiada todas las materias de preocupación local"92. En otras palabras,

86 COOK, W.W.: "What is the Police Power?", 7 Columbia Law Review, 1097, 322, p. 326. Por su parte, Novak observa: "más importante que la sanción formal constirucional [del poder de policía de los estados] son las raíces sustantivas del poder de regulación estatal en las tempranas nociones modernas de policía o Polizei". Novak, W.J., The People's Welfare..., cit,. p. 13. Véase también, en general, la Introducción de este libro. 113 U.S. 27, 30 (1885), énfasis añadido.

Barbier, 32 ,

123 U.S. 623,661 (1887).

Mugler, 662.

Pueden verse, entre otros, Patterson v. Kentucky, 97 U.S. 501, 504 (1878), y en el orden estatal, el tantas veces citado voto del Chief Justice Redfield en la Suprema Corte de Vermont en Thorpe v. Rutland, 27 Vt. 140, 149 (1855).

WILloughBy, W.W., The Constitutional Law of the United States, segunda edicion, Baker, Voorhis \& Co., New York, 1929, Vol. III, p. 1766. De modo parecido, Cook define el poder de policía como "el poder residual de gobierno, no clasificado, que la Constitución 
"poder de policía" era equivalente a la "policía interna" que el Primer Congreso Continental había reclamado para las colonias y que hemos encontrado en las discusiones que precedieron a la sanción de la constitución federal. Es decir que "el término [poder de policía] no se usaba para designar una rama o esfera de la autoridad legislativa de los Estados en especial; más bien se empleaba para deslindar la esfera de autoridad estatal de la del Gobierno General"93. Se trataba, se ha dicho con razón, de "una fórmula breve para la expresión de la idea federalista de las funciones del estado en un sistema federal"94. En teoría, para la Corte Suprema el poder de policía no incluía otras formas familiares de ejercicio de la soberanía de los estados que ya tenían nombres definidos como el "dominio eminente" 95 , la tributación y la administración de justicia 96. Sin embargo, en la práctica la Corte reconocía que las abarcaba o, más bien, que no podía separárselo de aquellas ${ }^{97}$.

Este significado amplio $[$ broad $]$ de poder de policía fue articulado de una manera especialmente clara por el Chief Justice Taney en su voto para la Corte [opinion for the Court] en los License Cases (1847). A la pregunta “¿Qué son los poderes de policía de un estado?”, respondía:

No son nada más ni nada menos que los poderes de gobierno propios de cada soberanía sobre la extensión de su dominio [...], es decir, el poder de soberanía, el poder de gobernar hombres y cosas dentro de los límites de sus dominios ${ }^{98}$.

Ya antes, el Justice Barbour había desplegado este concepto broad, usando la expresión "policía interna", en lugar de "poder de policía". En

de los Estados Unidos deposita en los respectivos Estados". COOK, W.W., "What is the Police Power?", cit,, p. 329. Barros anota que "el poder de policía como concepto del derecho constitucional estadounidense es sinónimo de la integridad del poder soberano de los estados que permanece después del reconocimiento constitucional de poderes limitados al gobierno federal". Barros, D. B., "The Police Power and the Takings Clause", 58, University of Miami Law Review, 2004, 471, p. 472.

93 Willoughiy, W.W., The Constitutional Law..., cit., Vol, III, p. 1766. Según Hastings, el poder de policía es un residuo que se obriene "después de haber sacado de los poderes generales de gobierno, primero los poderes que la convención de 1787 halló necesario poner en manos del gobierno general, y segundo otros poderes ordinariamente tenidos por soberanos que ya hubieran adquirido reconocimiento distintivo" (como el dominio eminente, el poder tributario y la administración de justicia). HASTINGS, W,G., "The Development of Law...", cit., p. 405.

94 Hastings, W.G., "The Development of Law...", cit., p. 372.

95 Se trata del poder de expropiación.

96 "En otras palabras, hemos tomado los poderes residuales de gobierno que los Estados tienen en nuestro sistema - la 'soberanía residual' de El Federalista-, los hemos clasificado y dado nombres específicos a algunas partes, por ejemplo, poder de tributación, de dominio eminente, etc., y después, quizá por falta de un nombre mejor, hemos llamado a lo restante "poder de policía". COOK, W.W., "What is the Police Power?", cit., p. 329. HASTINGS, W.G., "The Development of Law...", cit., p. 405.

985 Howard 504, $583(1847)$. 
Mayor of the City of New York v. Miln (1837), había sostenido, con cita de Brown v. Maryland:

Constituye no solo el derecho sino la carga y obligación solemne del estado promover la seguridad, felicidad y propiedad de su pueblo y proveer su bienestar general; todos estos poderes se relacionan con legislación meramente municipal o lo que, quizá, más propiamente podría llamarse policía interna $[\ldots]$ con relación a ellos la autoridad del estado es completa, incondicional y exclusiva ${ }^{99}$.

En suma, como consecuencia de la evolución histórica que hemos reseñado, "poder de policía" fue la frase elegida por los estadounidenses para expresar una noción central de su federalismo: que los estados retenían todo lo no delegado expresamente al gobierno federal. Ese todo al que se refiere la doctrina de los poderes delegados es el poder de policía. Es decir que el poder de policía estaba -y está- inescindiblemente unido al sistema federal.

Algunas autoridades estadounidenses, tanto jurisprudenciales como doctrinarias, encuentran al lado de esta definición amplia [broad] de poder de policía, una estrecha $[\text { narrow }]^{100}$. Según Willoughby, esta noción se habría comenzado a perfilar a fines del siglo XIX, cuando los estados intentaron justificar regulaciones locales, cuestionadas a la luz de la cláusula de la Constitución Federal que otorga al gobierno nacional la competencia para legislar en materia de comercio interestatal [Commerce Clause]. Se argumentaba que "las regulaciones en cuestión eran necesarias o razonablemente requeridas para la protección de la salud, la moralidad o el bienestar general de los ciudadanos de los Estados que las sancionaban [...]. Así, la frase poder de policía vino a referirse gradualmente, no a los poderes generales residuales de los Estados sino a su derecho a proveer y aplicar regulaciones razonables en favor de la moralidad, seguridad y conveniencia de sus habitantes"101, aun cuando el comercio interestatal se viera incidentalmente afectado ${ }^{102}$. De esta manera, a diferencia

99 11 Peters 102,139 (1837), énfasis agregado.

100 En la Argentina, Marcelo DE Jesús no parece reconocer este concepto narrow, cuando analiza la evolución del poder de policía en los Estados Unidos. DE Jesús, M.O., Análisis comparativo de la evolución de la doctrina del poder de policía en las Constituciones y en la jurisprudencia de los Estados Unidos de América y de la República Argentina (su influencia en la extensión del ámbito de los derechos fundamentales), Fundación Carlos Pellegrini, Buenos Aires, 1990 , ps. 10-28 y 46. Manuel García-Mansilla y Ricardo Ramírez Calvo me alertaron sobre la existencia de este trabajo.

101 Willoughby, W.W., The Constitutional Law..., cit., Vol. III, pp. 1766-1767.

102 En sentido parecido se expresa Freund: "El ejercicio del poder de policía para la protección de la seguridad, el orden y la moralidad (morals), constituye la policía en el sentido primario o más estrecho fnarrowerł del término. [...] Es el poder de policía en este sentido más estrecho del término (in this narrower sense of the term) lo que la Corte Suprema de 
de lo que había ocurrido hasta entonces, el poder de policía comenzó a designar también cierto campo o rama de la actividad legislativa - la destinada a restringir derechos por razones de moralidad, seguridad y salud públicas.

Un caso que ilustra bien esta concepción del poder de policía es Mugler v. Kansas (1887), en el que se había cuestionado la validez de una ley de Kansas que prohibía la producción y venta de bebidas alcohólicas. La Corte Suprema reconoció la competencia de la legislatura estatal para promover la moral, la salud y la seguridad públicas, y la fundó en el poder de policía:

¿A quién o a qué autoridad le corresponde determinar si la fabricación de ciertas bebidas destinadas ya sea al consumo general o al uso personal del fabricante afectan dañosamente al público? [...]. Bajo nuestro sistema de gobierno ello se halla a resguardo de la rama legislativa del gobierno. Corresponde a ese departamento ejercitar los que son conocidos como los poderes de policía del Estado y determinar, primariamente, qué medidas son apropiadas o necesarias para la protección de la moral pública [public morals], la salud pública o la seguridad pública ${ }^{103}$.

Como puede verse, esta noción estrecha [narrow] de poder de policía se diferencia de la original amplia $[b r o a d]$ en que aquella hace referencia a ciertas materias o contenidos (moral, salud, seguridad públicas; a veces se agrega bienestar público). En cambio el concepto amplio es de naturaleza residual y abarca todo lo no delegado (con la salvedad hecha de que en teoría no incluye el dominio eminente, la tributación y otros poderes retenidos por los estados pero que tienen ya nombres propios). De todas maneras, los dos conceptos, broad y narrow, tienen en común la referencia a los estados, ya que en ambos casos se trata de un poder retenido por ellos y no delegado al gobierno federal.

La definición del poder de policía por referencia a materias o contenidos tuvo especial vigencia durante la llamada "Era Lochner" ${ }^{104}$, durante

los Estados Unidos concede, en principio, a los estados, aun en los casos en que su ejercicio afecta el comercio interestatal y exterior". Freund, E., The Police Power..., cit., n. 10, p. 7. Véase también, Constitution of the United States of America. Analysis and Interpretation, séptima edición, U.S. Government Printing Office, Washington, 1973, pp. $1303-1318$.

103 123 U.S. 623, 660-61 (1887), énfasis añadido. Para una caracterización semejante del poder de policía, véase Powell v. Pennsylvania, 127 U.S. 678, 683 (1888), fallo en el que se sostuvo la validez de una ley estatal que prohibía la producción de oleomargarina.

104 Aunque tuvo sus antecedentes en fallos anteriores, como Allgeyer v. Lousiana, 165 U.S. 578 (1897), se considera que esta era comenzó en 1905 con Lochner v New York, 198 U.S. 45, y terminó a mediados de la década de 1930 con Nebbia v. New York, 291 U.S. 502 (1934) y West Coast Hotel v. Parrish, 300 U.S. 379 (1937). 
la cual la Corte usó intensamente la cláusula del debido proceso de la Constitución para invalidar controles sociales y económicos tales como el salario mínimo o la limitación de la jornada laboral, que habían sido impuestos por leyes estatales sancionadas en ejercicio del poder de policía. La definición estrecha [narrow] de este poder es una conclusión que se sigue del razonamiento de la Corte en Lochner y su progenie jurisprudencial. He aquí el argumento: i) el derecho x (v. gr. libertad de contratar) es una libertad protegida por la cláusula del debido proceso de la Enmienda XIV; ii) una invasión de dicha libertad solo puede justificarse en virtud de un apropiado ejercicio del poder de policía; iii) el poder de policía se ejerce apropiadamente solamente cuando se usa para ciertos abjetivos limitados, frecuentemente descriptos como la promoción de la salud, la seguridad y la moral públicas ${ }^{105}$.

Sin embargo, incluso durante la Era Lochner la Corte Suprema afirmó la coexistencia de los conceptos broad y narrow de poder de policía. Así, en 1907 la Corte declaró que los poderes de cada estado nunca entregados al gobierno general "pueden ser ejercidos no solo en pro de la salud pública, la moral pública y la seguridad pública, sino también para el bien general o común, para el bienestar, confort y buen orden de la gente"106. Y, más explícitamente, en 1919 afirmó que "las palabras 'poder de policía' son susceptibles de dos significados: uno comprehensivo (comprehensive), que abarca sustancialmente todo el campo de la autoridad estatal, y otro más estrecho (narrower) que incluye solamente el poder del estado para ocuparse de la salud, la seguridad y la moralidad de la gente" 107 .

Esta convivencia del concepto amplio y el estrecho se mantiene hasta la actualidad, como lo muestran fallos relativamente recientes. En Barnes v. Glen Theatre (1991) una pluralidad de la Corte afirmó: "El tradicional poder de policía de los Estados es definido como la autoridad para proveer lo conducente a la salud, la seguridad y la moral públicas, y hemos sostenido tal fundamento para la legislación"108. Por su parte, en United States v. Lopez (1995) la mayoría del Tribunal recordó la doctrina de los

105 Como explican Stone et al., si la Corte de la Era Lochner creía que la verdadera razón de una regulación era "la protección de la salud, la seguridad o la moral del público general, correspondía convalidar la ley. Pero si la Corte percibía la ley en cuestión como un esfuerzo para reajustar el mercado a favor de una de las partes de un contrato, lo más probable era que la declarase nula". STONE et al., Constitutional Law, tercera edición, Little, Brown, and Company, Boston, 1996, p. 831.

106 Western Turf Association v. Hyman Greenberg, 204 U.S. 359, 363 (1907).

107 Dakota Central Telephone Co. v. South Dakota ex rel. Payne, 250 U.S. 163, 185-186 (1919).

108501 U.S. 560, 569 (1991), voto de la pluralidad, citas intermedias omitidas. El caso está publicado en español en la revista El Derecho (1992) 148-594, con notas de G. J. BIDART CAMPOS y W. F. CARNOTA. 
poderes delegados y la noción original del poder de policía como soberanía residual:

Comenzamos con primeros principios. La Constitución crea un Gobierno Federal de poderes enumerados. Véase el Artículo I, Sección 8. Como escribió James Madison, "[1]os poderes delegados al gobierno federal por la Constitución propuesta son pocos y definidos. Los que permanecen en los gobiernos de los Estados son numerosos e indefinidos"109.

La Corte en Lopez concluyó, con palabras que ya he citado, afirmando que "[la] Constitución [no otorga] al Congreso un poder de policía plenario que autorizaría la sanción de cualquier tipo de legislación" Estamos, nuevamente, frente al concepto broad de poder de policía.

\section{E) CONCLUSIÓN}

Hemos repasado la historia constitucional estadounidense para ver cómo nació y se desarrolló el poder de policía. Ello nos llevó a la idea de policía y a sus antecedentes en Vattel, Blackstone y otros pensadores de la Europa ilustrada. Ya en Estados Unidos, la policía se transformó en poder de policía y sobrevive hasta hoy con este nombre, con los significados amplio [broad $]$ y estrecho [narrow $]$ que hemos observado.

Puestos a mirar la realidad argentina a la luz del experimento constitucional estadounidense, que tanto influyó en nuestro documento fundacional, surgen diversos interrogantes. ¿Es el poder de policía en la Argentina, al igual que en los Estados Unidos, un elemento esencial del federalismo? ¿Cómo se relacionan las definiciones broad y narrow que encontramos en los Estados Unidos con las correspondientes concepciones "amplia" y "estrecha" de las que hablan nuestros teóricos del derecho constitucional ${ }^{111}$ ? ¿Cómo debe entenderse, dentro del contexto analizado en este trabajo, la postulada supresión de la noción de poder de policía ${ }^{112}$ ? Es mi esperanza que las páginas precedentes puedan servir para iluminar estas y otras preguntas fundamentales del derecho público argentino.

\footnotetext{
109514 U.S. 549, 552 (1995), con cita del $\mathrm{N}^{\circ} 45$ de El Federalista.

110 United States v. Lopez, 514 U.S. 549, 566 (1995), voto de la mayoría.

111 Véase, por todos, BIDART CAMPos, G.J., Derecho Constitucional, Ediar, Buenos Aires, 1966, Vol. II, p. 548; Manual de la Constitución Reformada, Ediar, Buenos Aires, 1998, Vol. II, p. 345 .

112 Véase la sugerente argumentación de Gordil.o, A., Tratado de Derecho Administrativo, segunda edición, Fundación de Derecho Administrativo, Buenos Aires, 1998, Tomo 2, Cap. V, anticipada en 1960 en su arrículo "La crisis del poder de policía", recogido en Estudios de Derecho Administrativo, Perrot, Buenos Aires, 1963.
} 


\section{BIBLIOGRAFÍA}

- Ayto, J.: Dictionary of Word Origins, Arcade Publishing, Little, Brown, and Company, New York, 1990.

- BARnetT, R.E.; "The Proper Scope of the Police Power", 79, Notre Dame Law Review, 2004.

- Barros, D.B.: "The Police Power and the Takings Clause", 58, University of Miami Law Review, 2004.

- Bidart Campos, G.J.: Derecho Constitucional, Ediar, Buenos Aires, 1966, Vol. II.

- Bidart Campos, G.J.: Manual de la Constitución Reformada, Ediar, Buenos Aires, 1998, Vol. II.

- Blackstone, Sir W.: Commentaries on the Laws of England, edición facsímil del original de 1765-1769, University of Chicago Press, Chicago, 1979, Vol. I.

- Blackstone, Sir W.: Commentaries on the Laws of England, octava edición, W.G. Hammond, Bancroft-Whitney Company, San Francisco, 1890, Vol. IV.

- Cabanellas de las Cuevas, G., y Hoague, E.C.: Diccionario Jurídico Inglés-Español, Heliasta, Buenos Aires, 1993.

- Chambers Dictionary of Etymology: Chambers, Edinburgo, 1988.

- Constitution of the United States of America. Analysis And INTERPRETATION: séptima edición, U.S. Government Printing Office, Washington, 1973.

- CoOK, W.W: "What is the Police Power?", 7, en Columbia Law Review, 1907.

- Corominas, J., y Pascual, J.A.: Diccionario Crítico Etimológico Castellano e Hispánico, Gredos, Madrid, 1981, Vol. IV.

- CORWIN, E.S.: Liberty against Government. The Rise, Flowering and Decline of a Famous Juridical Concept, Louisiana State University Press, Baton Rouge, 1948.

- Crosskey, W.W.: Politics and the Constitution in the History of the United States, The University of Chicago Press, Chicago, 1953, Vol. I.

- De Jesús, M.O.: Análisis comparativo de la evolución de la doctrina del poder de policía en las Constituciones y en la jurisprudencia de los Estados Unidos de América y de la República Argentina (su influencia en la extensión del ámbito de los derechos fundamentales), Fundación Carlos Pellegrini, Buenos Aires, 1990.

- Diccionario DE AUTORIDADES: edición facsímil de la edición original de 1726-1737, Gredos, Madrid, 1976, Vol. III.

- El Federalista: Fondo de Cultura Económica, México, 1994.

- ERskine, J.: An Institute of the Law of Scotland, impresa por J. Bell en Addison's Head, Edinburgo, 1773. 
- Evans, L.B.: Leading Cases on American Constitutional Law, Callaghan \& Company, Chicago, 1925.

- FENwICK, C.G.: "The Authority of Vattel", 7, en The American Political Science Review, 1913.

- FinNis, J.M.: Ley Natural y Derechos Naturales, Abeledo-Perrot, Buenos Aires, 2000.

- Freund, E.: Standards of American Legislation. An Estimate of Restrictive and Constructive Factors, The University of Chicago Press, Chicago, 1917.

- Freund, E.: The Police Power. Public Policy and Constitutional Rights, reimpresión del original de 1904, Arno Press, New York, 1976.

- García-Mansilla, M.J., y Ramírez Calvo, R.: "James Madison y la Constitución Nacional", en Homenaje a la Constitución Nacional de 1853 en el sesquicentenario de su sanción, Instituto Urquiza de Estudios Históricos-Universidad de Belgrano, Buenos Aires, 2003.

- George, R.P.: "The Concept of Public Morality", 45, American Journal of Jurisprudence, 2000.

- Gordillo, A.: Tratado de Derecho Administrativo, segunda edición, Fundación de Derecho Administrativo, Buenos Aires, 1998, Tomo 2.

- Gordillo, A.: "La crisis del poder de policía", en Estudios de Derecho Administrativo, Perrot, Buenos Aires, 1963.

- Hastings, W.G.: "The Development of Law as Illustrated by the Decisions Relating to the Police Power of the State", en Proceedings of the American Philosophical Society, vol. XXXIX, 1999.

- Journals of the Continental Congress (1774-1789): edición a cargo de W.C. Ford et al., Government Printing Office, Washington, 1904-1937, Vol. I.

- KAMES, Lord H. H.: Statute Law of Scotland Abridged. With Historical Notes, impresa por Sands et al. para A. Kincald y A. Donaldson, Edinburgo, 1757.

- Kelly, J.M.: A Short History of Western Legal Theory, Clarendon Press, Oxford, 1992.

- Kent, J.: Commentaries on American Law, undécima edición, editada por G.F. Comstock, Little, Brown, and Company, Boston, 1866, Vol. II.

- Lapradelle, A. de: "Introduction", en Brown Scott, J. (ed.), The Classics of International Law. Le Droit des Gens, ou Principes de la Loi Naturelle, appliqués à la Conduite et aux Affaires des Nations et des Souverains, by E. de Vattel. Volume III, Carnegie Institution of Washington, Washington, 1916.

- Legarre, S.: "Los orígenes históricos del poder de policía", en El Derecho, 1999.

- Legarre, S.: "El poder de policía en la historia, la jurisprudencia y la doctrina", en La Ley, 2000-A-999. 
- Legarre, S.: Poder de Policía y Moralidad Pública. Fundamentos y Aplicaciones, Ábaco, Buenos Aires, 2004.

- MackenzIE, Sir G.: The Institutions of the Law of Scotland, cuarta edición, impresa por J. Watson para J. Vallange, Edinburgo, 1706.

- Nolan, D.R.: "Sir William Blackstone and the New American Republic: a Study of Intellectual Impact", 51, New York University Law Review, 1976.

- Notes of Debates in the Federal Convention of 1787 ReporTED By JAMES MAdison: W. W. Norton, New York, 1966.

- Novak, W.J.: The People's Welfare. Law and Regulation in NineteenthCentury America, The University of North Carolina Press, Chapel Hill, 1996.

- Oxford English Dictionary: segunda edición, Clarendon Press, Oxford, 1989.

- Scheiber, H.: "State Police Power", en Levy, L., et al., Encyclopaedia of the American Constitution, MacMillan Publishing Company, New York, 1986, Vol. IV.

- Smith, A.: Lectures on Jurisprudence, edición a cargo de R. L. Meek, D.D. Raphael y P.G. Stein, Clarendon Press, Oxford, 1978.

- Stimson, F.J.: The Law of the Federal and State Constitutions of the United States, Boston Book Company, Boston, 1908.

- The Records of the Federal Convention of 1787: 1937 Revised Edition, a cargo de M. Farrand, Yale University Press, New Haven, 1966, Vol. 2.

- Thorpe, F.N.: The Federal and State Constitutions, Colonial Charters, and other Organic Laws of the States, Territories, and Colonies now or beretofore forming the United Sates of America, Government Printing Office, Washington, 1909.

- Tiedeman, C.G.: A Treatise on State and Federal Control of Persons and Property in the United States considered from both a civil and criminal standpoint, The F.H. Thomas Law Book Co., St. Louis, Missouri, 1900.

- Tribe, L.H.: American Constitutional Law, tercera edición, The Foundation Press, New York, 2000, Vol. I.

- VATTEL, E. de: The Law of Nations or Principles of the Law of Nature: applied to the Conduct and Affairs of Nations and Sovereigns, Newbery et al., Londres, 1759-1760.

- Willoughby, W.W.: The Constitutional Law of the United States, segunda edición, Baker, Voorhis \& Co., New York, 1929, Vol. III. 